\title{
On-bed Monitoring for Range of Motion Exercises with a Pressure Sensitive Bedsheet
}

\author{
Jason J. Liu, Ming-Chun Huang, Wenyao Xu, Nabil Alshurafa, Majid Sarrafzadeh \\ Wireless Health Institute, Department of Computer Science \\ University of California, Los Angeles \\ Email: \{jasonliu, mingchuh, wenyao,nabil, majid\}@cs.ucla.edu
}

\begin{abstract}
This paper presents the design of an on-bed rehabilitation exercise monitoring system that utilizes a high density sensor bedsheet to evaluate active range of motion exercises. We propose and develop a novel framework to analyze the progression of pressure image sequences using manifold learning. The image sequences are reduced to a low dimensional subspace that can be measured against expected prior data for each of the rehabilitation exercises. We also present a metric to compare manifold similarities. Our experimental results on five on-bed exercises show that this system can accurately track compliance of patients to prescribed treatment programs. It allows physical therapists to evaluate how well patients adhere to the rehabilitation exercises. The system is convenient to setup, unobtrusive, and can be used for reliable, long term monitoring.
\end{abstract}

Keywords—physical rehabilitation; range of motion; pressure images; manifold learning; local linear embedding.

\section{INTRODUCTION}

Physical rehabilitation is a treatment program designed to help patients return to normal health following surgery or illness. In most cases, the aim is to improve muscular strength and range of motion. Traditionally, rehabilitation programs are carried out in hospitals or therapy treatment centers, where trained physical therapists provide instruction and monitor patients.

\section{A. Background}

Physical rehabilitation is a proven method that is well recognized to provide lasting benefits to patients [1], [2]. For example, Johns Hopkins Hospital has reported up to $22 \%$ reduction in ICU length of stays and reductions in net financial costs due specifically to the inclusion of early physical rehabilitation programs [3]. Patients undergoing physical rehabilitation follow the exercises assigned by physical therapists on a regular or semi-regular basis. Physical therapists need to manually monitor and evaluate the rehabilitation process to check that the progress of the patient goes according to expectation. As a result, the cost of monitoring rehabilitative exercises is large and the recovery progress of each patient is difficult to quantize.

There are four types of physical rehabilitation treatments or range of motion (ROM) exercises: (a) passive ROM, where a physical therapist applies external force to move the subject's body; (b) active assisted ROM, where the subject moves with assistance from a therapist; (c) active ROM, where the subject moves by himself; and (d) self-assisted ROM is when the subject moves himself but can, for instance, use his arms to extend the motion of his rehabilitated legs. This work targets both active and self-assisted range of motion (ROM) exercises where the subjects are able to carry out the exercises without assistance from physical therapists.

\section{B. Related Work}

Zhou and Hu's survey of human motion tracking systems that relate to rehabilitation of stroke sufferers offers a list of technological approaches [4]. Exercise machines, such as treadmills, or even robotic devices [5] have been used to guide patients to build their strength. Other techniques such as visual tracking of body posture has relied mainly on marker systems placed on the body [6]. This is a limitation that marker-free systems try to overcome by building 2-D [7] or 3-D models of the human body [8]. Non-visual methods that use inertial sensors have also been researched [9]. Huang et al. combined inertial sensors and visual camera tracking to monitor both (fine-grain) finger and (course-grain) hand movements for upper extremities rehabilitation [10].

This paper deals with patients who are bed-ridden or are restricted to motions on bed. There are current research approaches that focus on detection of posture changes on bed. Nakajima et al. analyzed posture change using real-time video image sequences to extract optical flow information [11]. Jones et al. used a 24 pressure sensor array to identify movement times and hence evaluated sleep restlessness [12]. Adami et al. used 4 load cell sensors with a sampling rate of $200 \mathrm{~Hz}$ to analyze the time varying waveforms when patients move on bed [13].

These previous methods show accurate detection of posture movement, i.e. the existence of body movement or posture changes, however they do not target the recognition of actual posture. Foubert et al. developed a system to detect transitions between sitting and lying postures using large pressure sensor arrays placed under the mattress [14]. Sleep posture recognition has been successful in analyzing images using principle component analysis (PCA) [15], as well as using statistical feature extraction [16]. Harada et al. investigated body posture tracking and used generative models of human body pressure to match the patient's pressure distribution [17]. Our previous work in this field investigated static posture recognition [18], however, the aim of this current research is toward transitional and dynamic motion of on-bed exercises. 


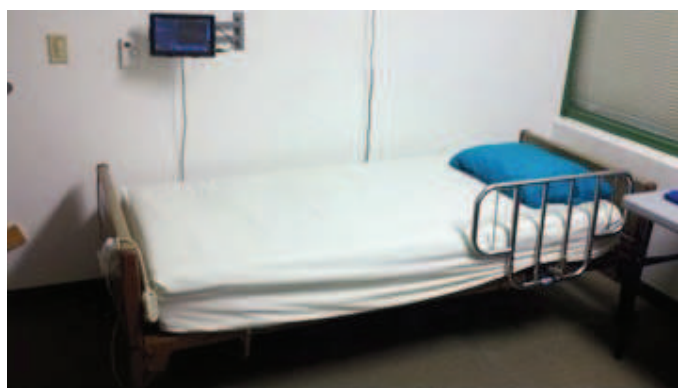

Fig. 1. Bedsheet Prototype

The remainder of the paper is organized as follows. Section II describes the overall design of this monitoring system that incorporates a pressure sensitive bedsheet. Section III describes the algorithmic process of on-bed rehabilitation exercise monitoring using a dimension reduction method on body pressure image sequences. Experimental set up and results are given in Section IV. Finally, future work and conclusion are discussed in Section V.

\section{SYSTEM OVERVIEW}

This paper proposes a method to monitor patient coherence to therapy specifically targeting a range of on-bed rehabilitation exercises. In order to show this, we propose a method to recognize different exercises. This system would also enable the physical therapist to track progress not only within clinical settings but also within home-based environments.

Our system design consists of a high density sensor bedsheet and a connected tablet which collects the pressure image sequences. The tablet analyses the data and transmits the results through wired or wireless communication to a monitoring station. A subject lies on the bedsheet and follows the instruction given by physical therapists. The tablet along with the bed sheet can pre-store the treatment scripts in its persistent storage and play the scripted text visually and vocally. Our system will monitor and infer the patient body structure by analyzing the pressure map sequences. If the subject does not follow the preset goals, warning messages are displayed.

\section{A. Bedsheet design}

Figure 1 shows the prototype of the bedsheet system. The system consists of three components: a $64 \times 128$ pressure sensor array, a data sampling unit, and a tablet for data analysis and storage. The sensor array is based on eTextile material which is fiber-based yarn coated with piezoelectric polymer [19], [20]. The initial resistance of the eTextile material is high. As external force is applied to the surfaces of the material, the eTextile fibers are squeezed together and, due to its pressure sensitive characteristics, the electrical resistance decreases in that region.

The bedsheet has a three-layer sandwiched structure. The top layer is regular fabric that is coated with 64 parallel conductive lines. The middle layer is the eTextile material and the bottom layer has 128 conductive lines arranged perpendicular to the top 64 lines. At each intersection of conductive lines, the structure forms a pressure sensitive resistor. There are effectively 8192 pressure sensors in total.

\section{B. Algorithm Overview}

Exercise recognition uses a subject's pre-recorded training data to match exercises under testing. The training data consists of samples of on-bed exercises that are analyzed to produce a low dimensional representation from the original high resolution pressure images. When new exercise data is recorded, it is mapped to the same low dimensional representation and matched to the closest exercise. This process is described in detail in the following section.

\section{Algorithmic Framework FOr EXERCiSE MONITORING}

This section details the algorithmic framework of the onbed patient exercise recognition. Figure 2 shows the three main steps: pre-processing of the pressure image; dimension reduction via manifold learning; and activity recognition using manifold matching.

\section{A. Pre-processing}

The pre-processing of the raw pressure images is required so that the image sequences can be standardized in such a way to enable successful recognition. The raw images contain noise and artifacts that affect recognition, and pre-processing mitigates the side effects as much as possible.

Firstly, the subject can be located anywhere on the bedsheet, so to correct this, the images are aligned to a common center of mass and relocated to the center of the image. A smoothing filter of a symmetric $5 \times 5$ unit normal distribution is applied. This smoothing minimizes the effect of noise in the pressure map. The images are normalized so that the sum of pixel weights is one. This step attempts to counteract the affects for the differing body mass.

\section{B. Dimension Reduction using Manifold Learning}

Our method to map the image sequence $X$ to a low dimensional space is based on the Local Linear Embedding (LLE) framework by Saul and Roweis [21], which has various applications in machine learning systems [22]. LLE is an unsupervised algorithm that reconstructs the global data nonlinearly while preserving local linearity. After the computation, similar images will be clustered within the low dimensional manifold. In general, there are three steps in the algorithm, which will be described in the following.

1) k-Nearest Neighbor Searching: The first step is to search $\mathrm{k}$-nearest neighbors for each image. In the searching process, we use Euclidean distance to evaluate the similarity between images. There are two ways to determine group size $\mathrm{k}$ in the searching procedure. One is using fixed integer. The other way is to identify the neighborhood by a threshold value in distance metrics. In this method, any image within a given distance will be recognized as a neighbor. Normally the topology of embedding will be well-preserved over a range of neighborhood sizes. For this work, we searched for the 30 nearest neighbors of each image. 


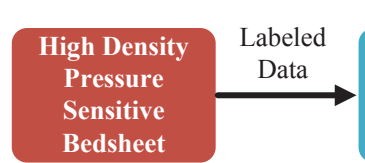

Raw Pressure Images

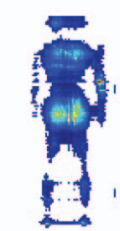

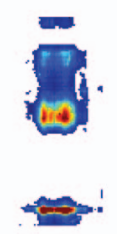

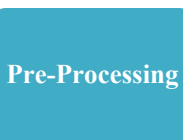

Testing Process

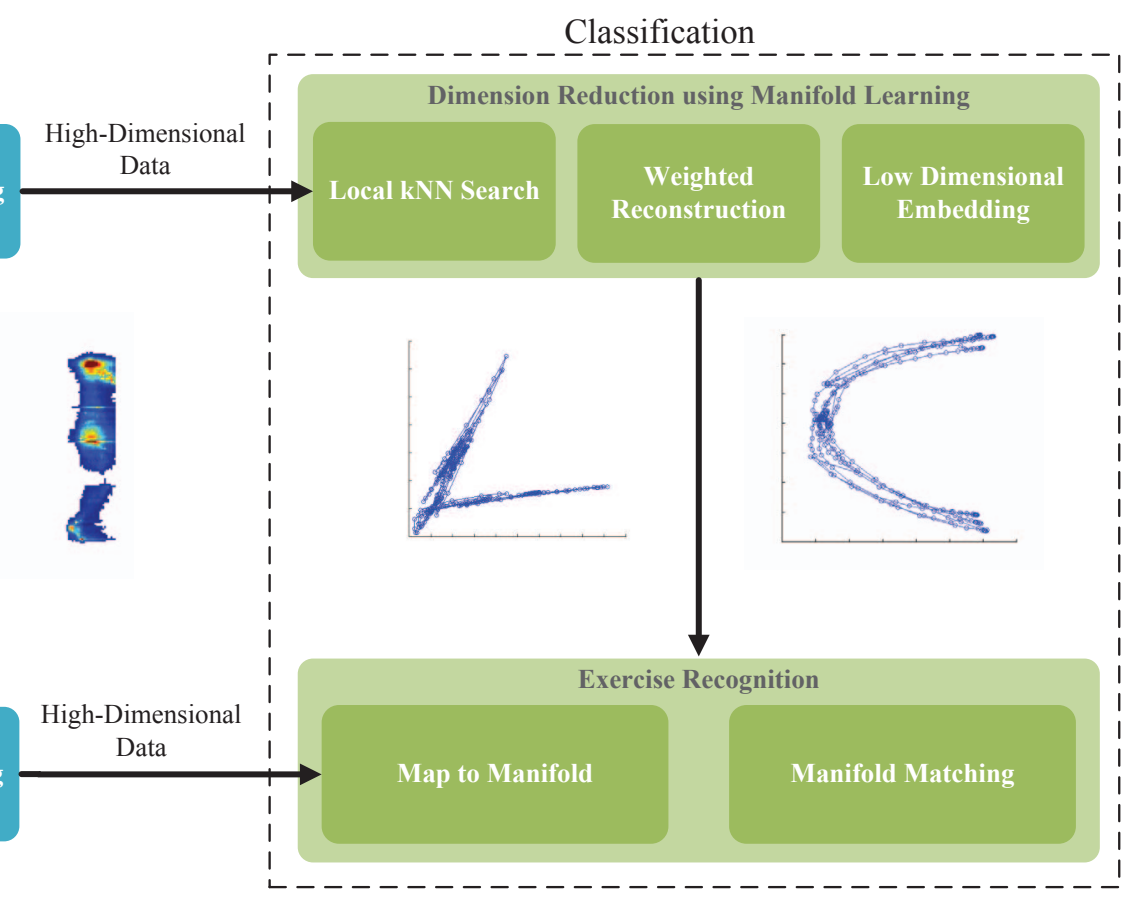

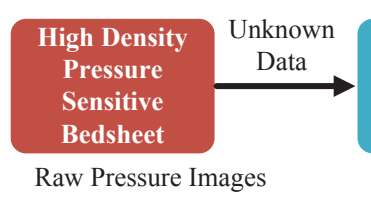

Raw Pressure Images

Fig. 2. Process Flow for On-bed Exercise Monitoring

2) Weighted Reconstruction With Nearest Neighbors: The second step is to reconstruct a sample image using its nearest neighbors. Assume that an arbitrary image $x$ has k-nearest neighbors $x_{i}$. Then $x$ can ideally be represented as a linear combination of its neighbors. In general, an exact reconstruction will not be found, so a reconstruction error $e$ can be formulated as:

$$
e=\left\|x-\sum_{i=1}^{k} w_{i} x_{i}\right\|,
$$

where $w_{i}$ denotes the reconstruction weight for the neighbor $x_{i}$. The optimization process minimizes the reconstruction error of all images by setting the weight $w_{i}$ values. There are two attributes of the problem to ensure it is well-imposed: (1) exclusiveness: the weight $w_{i}$ of $x$ is zero if $x_{i}$ is not in the nearest neighbor list of $x$; (2) normalization: the sum of the weights of nearest neighbors should be 1 . Therefore, we can rewrite the problem for all images:

$$
E=\sum_{j=1}^{N}\left\|x_{j}-\sum_{i=i}^{N} w_{i j} x_{i j}\right\| .
$$

We can see that Equation (2) represents the reconstruction problem and has a closed least square solution, where the weights $w_{i j}$ can be solved efficiently [21].

3) Low Dimensional Embedding Construction: The third step is to construct the corresponding embedding in a low dimensional space. Based on the calculation results from the second step, the intrinsic geometrical structure of each local cluster is characterized by $w_{i j}$. We assume that the neighborhood relation in high dimensional space should be preserved in low dimensional space, i.e. within a manifold. Based on this assumption, the embedding process is to search for the low dimensional representation $y$ of $x$ by minimizing the following error $E^{\prime}$ :

$$
E^{\prime}=\sum_{j=1}^{N}\left\|y_{j}-\sum_{i=i}^{N} w_{i j} y_{i j}\right\|,
$$

where $y_{j}$ are the corresponding points in the low dimensional manifold. We note that Equation (3) is in a quadratic form and the embedding optimization process is efficiently solvable. Furthermore, all the manifold points $y_{i}$ will be computed globally and simultaneously, and no local optima will affect the construction result.

Equation (2) indicates that the low dimensional construction is only based on the locality of the high dimension data. This means that the computed manifold $y_{i}$ can be translated with an arbitrary displacement without affecting Equation (3). Moreover, LLE states the computed manifold $y_{i}$ can be rotated by an arbitrary angle without affecting Equation (3) too. This geometric attribute can be represented and formulated in the following two equations:

$$
\begin{gathered}
\sum_{i=1}^{N} y_{i}=0 \\
\frac{1}{N} \sum_{i=1}^{N} y_{i} \cdot y_{i}=1 .
\end{gathered}
$$


Therefore, manifold construction problem becomes an eigenvalue problem [21], in which we select the matrix rank to have the desired manifold dimension.

\section{Exercise Recognition using Manifold Matching}

1) Map input to manifold: Once the training data has been reduced in dimensionality to its corresponding low dimensional form, we can evaluate the process using new test data against the training data. The testing data needs to be converted into manifold form. Note that it is possible to run the whole LLE algorithm again on the combined testing data and training data in order to find the low dimensional representation of the test data, however this would be slow in a real system.

Instead, a portion of the algorithm need only be executed [21], [23]. Given a new test image $\hat{x}$, we wish to find its low dimensional representation, $\hat{y}$. To do so, the weights $w_{i}$ are computed from the $\mathrm{k}$ nearest neighbors of $\hat{x}$ in the training set, $x_{i}$. This is again the least squares solution to minimize

$$
\left\|\hat{x}-\sum_{i=1}^{k} w_{i} x_{i}\right\|
$$

with the constraint $\sum_{i=1}^{k} w_{i}=1$. Since the corresponding low dimensional co-ordinates of $x_{i}$ are known during the training phase, we can construct the resultant embedded co-ordinates for $\hat{y}$ using the same weights:

$$
\hat{y}=\sum_{i=1}^{k} w_{i} y_{i}
$$

where $y_{i}$ are the corresponding embedded points of $x_{i}$.

2) Manifold Matching: Exercise tracking involves checking how well the test testing data follows the trajectory of a given exercise manifold. We can compare trajectories using a similar idea to the Hausdorff distance. The distance of a point to a manifold is equal to the shortest Euclidean distance to any point in the manifold. The similarity of two manifolds is the mean of the point distances of all the points of one manifold, $M_{1}$ to the other manifold, $M_{2}$. This is expressed as

$$
s\left(M_{1}, M_{2}\right)=\frac{1}{T_{M_{1}}} \sum_{i=1}^{T_{M_{1}}} \min _{1 \leq j \leq T_{M_{2}}}\left\|M_{1}(i)-M_{2}(j)\right\|,
$$

where $T_{M_{1}}$ and $T_{M_{2}}$ are the number of points in each manifold. This metric allows manifolds of different lengths to be compared since different subjects take different times to perform each activity. Since the Hausdorff metric is not symmetric, we can take the following sum as the manifold matching metric,

$$
d\left(M_{1}, M_{2}\right)=s\left(M_{1}, M_{2}\right)+s\left(M_{2}, M_{1}\right)
$$

So, to measure how well a subject adheres to the prescribed exercise, the testing data is mapped to corresponding low dimensional embedding points that defines a manifold, then the manifold is measured against the expected exercise manifold.

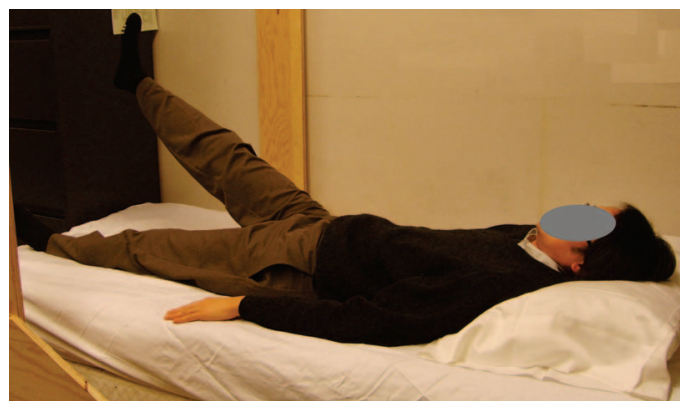

Fig. 3. Example of Leg Lift Exercise
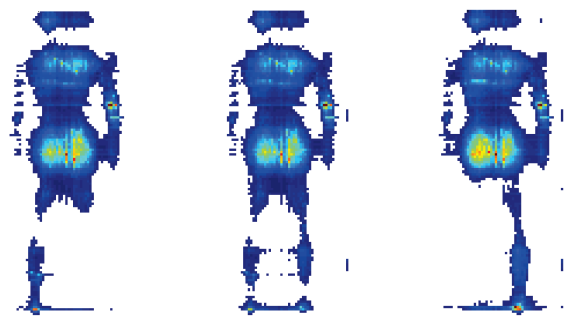

Fig. 4. Left and Right Leg Lift

\section{EXPERIMENTAL RESULTS}

\section{A. Experimental Setup}

The framework for exercise monitoring was evaluated on 10 subjects, 7 male subjects and 3 female subjects. The weight of the subjects ranged from $50 \mathrm{~kg}$ to $85 \mathrm{~kg}$, and height between $155 \mathrm{~cm}$ and $188 \mathrm{~cm}$. There were 5 selected on-bed exercises: alternating leg-lifts, head-lifts, alternating heel slides, alternating lateral rolls (lying on back to lying on side), and sit-ups. These exercises have been selected as being appropriate for on-bed monitoring [24]. In the training data collection, at least 5 sets of image sequences were recorded for each of the 5 on-bed exercises for each subject. Each image sequence comprises one exercise activity, e.g. one leg lift exercise activity includes lifting of the right leg followed by the left leg. The order of left and right does not matter in this system. Each image sequence of exercise activity contained at least 40 individual images. Variations in body, arm and leg positions were allowed.

The training data for each subject was combined and manifold learning was applied to generate the training manifolds

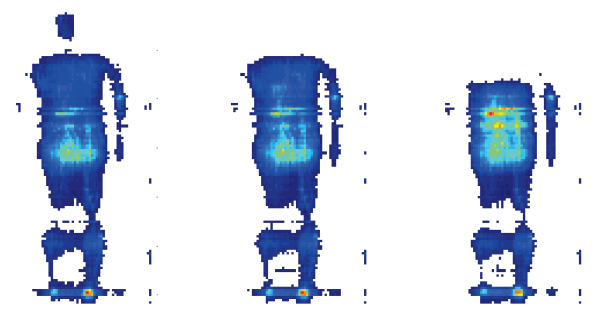

Fig. 5. Head Lift 
TABLE I. CONFUSION MATRIX

\begin{tabular}{|c|c|c|c|c|c|c|c|}
\hline & Leg Lift & Head Lift & Heel Slide & Lateral Roll & Sit Up & Total & Recall \\
\hline Leg Lift & 38 & 3 & 5 & 0 & 0 & 46 & $82.6 \%$ \\
Head Lift & 8 & 39 & 2 & 0 & 0 & 49 & $79.6 \%$ \\
Heel Slide & 7 & 0 & 54 & 0 & 0 & 61 & $88.5 \%$ \\
Lateral Roll & 0 & 0 & 0 & 44 & 0 & 44 & $100 \%$ \\
Sit Up & 0 & 0 & 0 & 1 & 56 & 57 & $98.2 \%$ \\
\hline Total & 53 & 42 & 61 & 45 & 56 & 257 & \\
\hline Precision & $71.7 \%$ & $92.9 \%$ & $88.5 \%$ & $97.8 \%$ & $100 \%$ & & \\
\hline
\end{tabular}

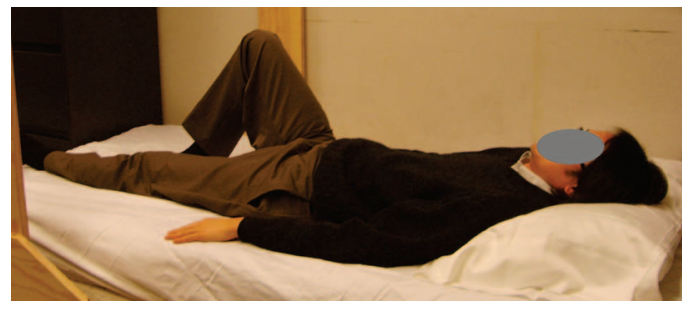

Fig. 6. Example of Heel Slide Exercise
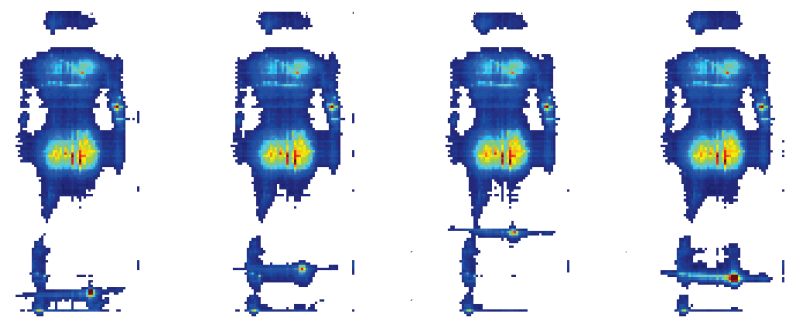

Fig. 7. Right Heel Slide

for the exercises. Testing was carried out by exercise activity and repeated for each of the exercise activities.

\section{B. Experimental Evaluation}

Table I shows recognition results for the 5 exercises in 10 subject dependent testing. Notably, the highest recognition rates are Lateral Rolls and Sit Ups. This can be expected since these exercises involve the greatest physical exertion and hence the greatest pressure image differences. The other three exercises exhibit a comparably lower rate of recognition due to more of a fine grain difference in the pressure image
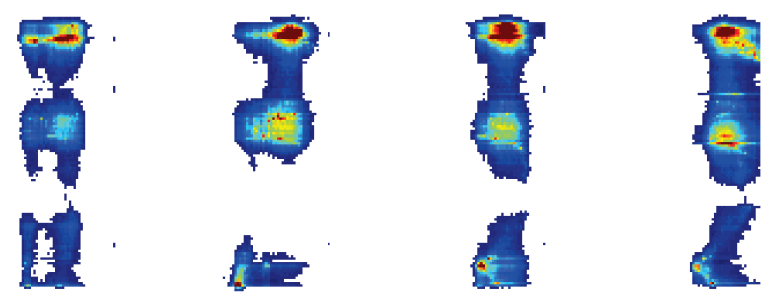

Fig. 8. Lateral Rolls sequences. The confusion matrix shows that there are the most misclassifications between Left Lifts and Heel Slides. By observing Figures 4 and 7, there are clear similarities in the pressure images.
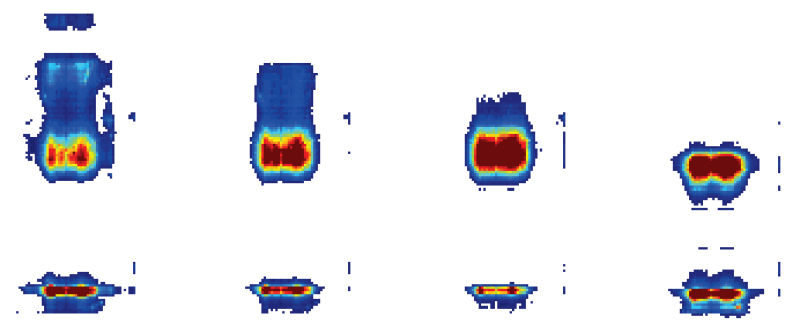

Fig. 9. Sit Up

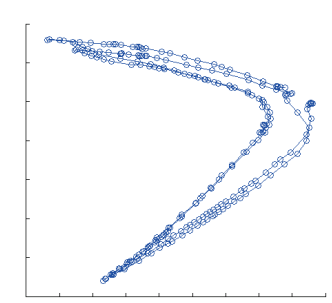

(a) Leg Lift

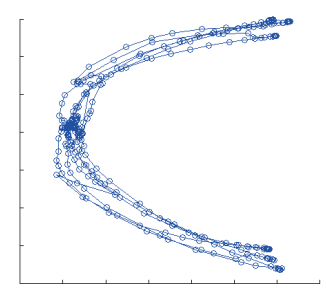

(c) Lateral Roll

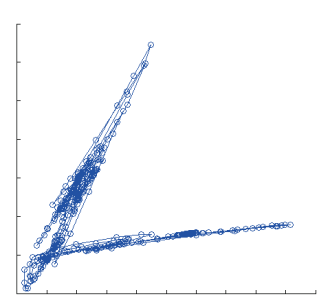

(b) Heel Slide

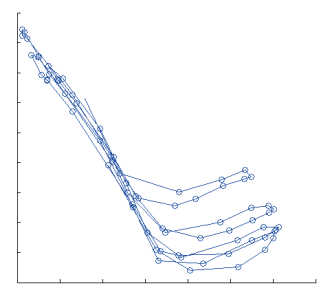

(d) Sit Up
Fig. 10. Samples of Exercise Manifolds

Figure 10 shows samples of the low dimensional visualization of manifolds for some of the exercises. Generally the shapes of the manifolds give an indication of the differences between the exercises. Using the Manifold Matching method, a quantified measurement of exercise recognition is performed.

Figure 11 shows samples of how head-lifts appear on a leglift manifold, and sit-up compared to lateral rolls. It is evident that the sample exercises can be discerned from each other. It 
is interesting to note in Figure 10(d) that the variations in sit ups can be seen.

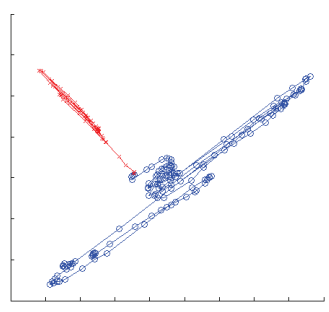

(a) Leg Lift (blue) vs Head Lift (red)

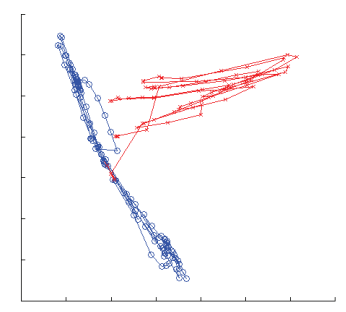

(b) Roll (blue) vs Sit up (red)
Fig. 11. Samples of Exercise Manifolds

The dimension reduction algorithm requires the data to be non-sparse, i.e. there must be sufficient sampling of pressure images to track motions. The current state of technology for pressure images of this resolution are 2-5 samples per second. Higher sampling rates can be achieved with the loss of image resolution.

\section{CONCLUSION}

This work presents an on-bed exercise monitoring system design that allows care-givers to track compliance to physical rehabilitation programs. This work also presents the novel use of a dimension reduction technique from pressure images to find intrinsic subspace representations of the data. We also evaluated a metric to match manifolds to enable quantified measurement of coherence to prescribed exercises.

Future work involves quantifying the performance of a given exercise with respect to a standard exercise model. Other future endeavors includes facilitating a system to work on chairs for sitting rehabilitative exercise, not only in clinical rooms or home-base care but also for cars or wheelchairs. 3D model reconstruction of patients from 2D pressure image is another goal that can be accomplished using the results of this research work.

\section{ACKNOWLEDGMENT}

The authors would like to thank Medisens Wireless Inc. for building and supplying the hardware.

\section{REFERENCES}

[1] A. L. Behrman and S. J. Harkema, "Physical rehabilitation as an agent for recovery after spinal cord injury," Physical Medicine and Rehab Clinics of North America, vol. 18, no. 2, pp. 183-202, 2007.

[2] M. Fransen, J. Crosbie, and J. Edmonds, "Physical therapy is effective for patients with osteoarthritis of the knee: a randomized controlled clinical trial." The Journal of Rheumatology, vol. 28, no. 1, pp. 156164, 2001.

[3] R. Lord, C. Mayhew, R. Korupolu, E. Mantheiy, M. Friedman, J. Palmer, and D. Needham, "ICU Early Physical Rehabilitation Programs: Financial Modeling of Cost Savings." Critical Care Medicine, Jan. 2013.

[4] H. Zhou and H. Hu, "Human motion tracking for rehabilitation - A survey," Biomedical Signal Proc. and Control, vol. 3, pp. 1-18, 2008.

[5] H. I. Krebs, B. T. Volpe, M. L. Aisen, and N. Hogan, "Increasing productivity and quality of care : Robot-aided neuro-rehabilitation," Rehabilitation Research \& Development, vol. 37, p. 639, Nov 2000.
[6] Y. Tao and H. Hu, "Buiding a visual tracking system for home-based rehabilitation," in In Proceedings of the 9th Chinese Automation and Computing Society Conference In the UK, 2003, pp. 343-348.

[7] R. Fablet and M. J. Black, "Automatic detection and tracking of human motion with a view-based representation," in Proceedings of the 7th European Conference on Computer Vision-Part I, 2002, pp. 476-491.

[8] Q. Delamarre and O. Faugeras, "3d articulated models and multiview tracking with physical forces," Computer Vision and Image Understanding, vol. 81, no. 3, pp. 328-357, 2001.

[9] E. Jovanov, A. Milenkovic, C. Otto, P. de Groen, B. Johnson, S. Warren, and G. Taibi, "A WBAN System for Ambulatory Monitoring of Physical Activity and Health Status: Applications and Challenges," in 27th Annual International Conference of the Engineering in Medicine and Biology Society, Jan. 2005, pp. 3810-3813.

[10] M.-C. Huang, W. Xu, Y. Su, B. Lange, C.-Y. Chang, and M. Sarrafzadeh, "Smartglove for upper extremities rehabilitative gaming assessment," in Proceedings of the 5th International Conference on Pervasive Technologies Related to Assistive Environments, 2012, pp. 20:1-20:4.

[11] K. Nakajima, Y. Matsumoto, and T. Tamura, "Development of real-time image sequence analysis for evaluating posture change and respiratory rate of subject in bed," Physiological Measurement, vol. 22, no. 3, p. N21, 2001.

[12] M. Jones, R. Goubran, and F. Knoefel, "Identifying movement onset times for a bed-based pressure sensor array," in Medical Measurement and Applications. IEEE International Workshop, April 2006, pp. 111114.

[13] A. M. Adami, M. Pavel, T. L. Hayes, and C. M. Singer, "Detection of movement in bed using unobtrusive load cell sensors," Transactions on Infomation Technology in Biomedicine, vol. 14, no. 2, pp. 481-490, Mar. 2010.

[14] N. Foubert, A. McKee, R. Goubran, and F. Knoefel, "Lying and sitting posture recognition and transition detection using a pressure sensor array," in Medical Measurements and Applications Proceedings (MeMeA), 2012 IEEE International Symposium on, May 2012, pp. 1-6.

[15] R. Yousefi, S. Ostadabbas, M. Faezipour, M. Farshbaf, M. Nourani, L. Tamil, and M. Pompeo, "Bed posture classification for pressure ulcer prevention," in Engineering in Medicine and Biology Society, Sept. 2011, pp. 7175-7178.

[16] C.-C. Hsia, Y.-W. Hung, Y.-H. Chiu, and C.-H. Kang, "Bayesian classification for bed posture detection based on kurtosis and skewness estimation," in e-health Networking, Applications and Services, HealthCom 10th International Conference, July 2008, pp. 165-168.

[17] T. Harada, T. Sato, and T. Mori, "Human motion tracking system based on skeleton and surface integration model using pressure sensors distribution bed," in Proc. Workshop on Human Motion, 2000, p. 99.

[18] J. J. Liu, W. Xu, M.-C. Huang, N. Alshurafa, and M. Sarrafzadeh, “A dense pressure sensitive bedsheet design for unobtrusive sleep posture monitoring," in IEEE International Conference on Pervasive Computing and Communications, Mar 2013.

[19] W. Xu, Z. Li, M.-C. Huang, N. Amini, and M. Sarrafzadeh, "eCushion: An eTextile Device for Sitting Posture Monitoring," in Body Sensor Networks (BSN), May 2011, pp. 194-199.

[20] W. Xu, M.-C. Huang, N. Amini, J. J. Liu, L. He, and M. Sarrafzadeh, "Smart insole: a wearable system for gait analysis," in Proceedings of the 5th International Conference on PErvasive Technologies Related to Assistive Environments, ser. PETRA '12, 2012, pp. 18:1-18:4.

[21] L. Saul and S. Roweis, "Think globally, fit locally: unsupervised learning of low dimensional manifolds," Journal of Machine Learning Research, vol. 4, pp. 119-155, Dec. 2003.

[22] Z. Li, W. Xu, A. Huang, and M. Sarrafzadeh, "Dimensionality reduction for anomaly detection in electrocardiography: A manifold approach," in Wearable and Implantable Body Sensor Networks (BSN), 2012 Ninth International Conference, 2012, pp. 161-165.

[23] M. Zhang and A. Sawchuk, "Manifold learning and recognition of human activity using body-area sensors," in Machine Learning and Applications and Workshops (ICMLA), 10th International Conference, vol. 2, Dec. 2011, pp. 7-13.

[24] S. Nettina and L. W. . Wilkins, The Lippincott Manual of Nursing Practice. Lippincott Williams \& Wilkins, 2006. 